\title{
High incidence of thromboembolism in patients with chronic GVHD: association with severity of GVHD and donor-recipient $A B O$ blood group
}

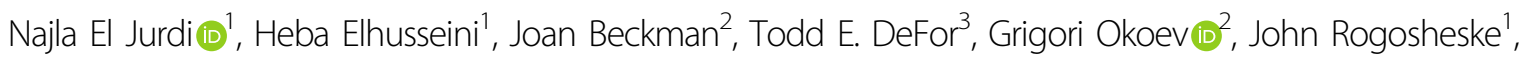

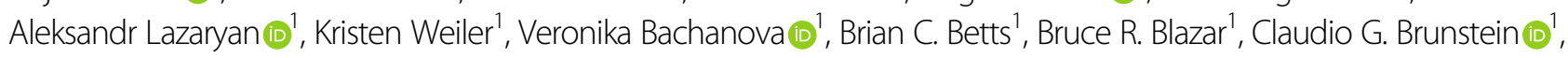
Fiona He (1)', Shernan G. Holtan', Murali Janakiram [1', Radhika Gangaraju4', Joseph Maakaron (1)', Margaret L. MacMillan (1)', Armin Rashidi', Erica D. Warlick', Smita Bhatia ${ }^{4}$, Gregory Vercellotti

Daniel J. Weisdorf(10 ${ }^{1}$ and Mukta Arora' ${ }^{1}$

\begin{abstract}
Chronic graft-versus-host disease (cGVHD) after allogeneic hematopoietic cell transplantation (HCT) is associated with systemic inflammation and endothelial dysfunction, increasing risk for thromboembolic events (TEE). In 145 adult recipients who developed CGVHD after a matched sibling or umbilical cord blood donor HCT from 2010 to 2018, 32 (22\%) developed at least 1 TEE event, and 14(10\%) developed 2 TEE events. The 5-year cumulative incidence of TEE was $22 \%(95 \% \mathrm{Cl}, 15-29 \%)$ with a median time from cGVHD to TEE of 234 days (range, 12-2050). Median time to the development of LE DVT or PE was 107 (range, 12-1925) compared to 450 days (range, 158-1300) for UE DVT. Cumulative incidence of TEE was 9\% (95\% Cl, $0-20 \%), 17 \%(95 \% \mathrm{Cl}, 9-25 \%)$, and $38 \%(95 \% \mathrm{Cl}, 22-55 \%)$ in those with mild, moderate, and severe GVHD, respectively. Higher risk for TEE was associated with CGVHD severity (hazard ratio [HR] 4.9, [95\% Cl, 1.1-22.0]; $p=0.03$ ), non-O-donor to recipient $\mathrm{ABO}$ match compared to O-donor to O-recipient match (HR 2.7, $[95 \% \mathrm{Cl}, 1.0-7.5] ; p=0.053)$, and personal history of coronary artery disease (HR 2.4, $[95 \% \mathrm{Cl}, 1.1-5.3] ; p=0.03)$. TEE was not associated with 2-year non-relapse mortality or 5-year overall survival.
\end{abstract}

\section{Key points}

- Patients with chronic GVHD after allogeneic hematopoietic cell transplantation are at high risk for thromboembolic events occurring years after diagnosis.

- More severe chronic GVHD, non-O donor-recipient ABO compared to O-O match and personal history of coronary artery disease are associated with higher risk of thromboembolic events.

Correspondence: Najla El Jurdi (neljurdi@umn.edu)

${ }^{1}$ Blood and Marrow Transplant Program, Department of Medicine, University of Minnesota, Minneapolis, MN, USA

2Division of Hematology, Oncology, and Transplantation, Department of Medicine, University of Minnesota, Minneapolis, MN, USA

Full list of author information is available at the end of the article

\section{Introduction}

Chronic graft-versus-host disease (cGVHD) occurs in up to half of allogeneic HCT recipients, limits the success of allogeneic hematopoietic cell transplantation (HCT), and remains the leading cause of non-relapse mortality (NRM) and morbidity among survivors. cGVHD is a multisystem syndrome involving dysregulated immunity, tissue inflammation and injury, with endothelial dysfunction

\section{(c) The Author(s) 2021}

(c) (i) Open Access This article is licensed under a Creative Commons Attribution 4.0 International License, which permits use, sharing, adaptation, distribution and reproduction in any medium or format, as long as you give appropriate credit to the original author(s) and the source, provide a link to the Creative Commons license, and indicate if changes were made. The images or other third party material in this article are included in the article's Creative Commons license, unless indicated otherwise in a credit line to the material. If material is not included in the article's Creative Commons license and your intended use is not permitted by statutory regulation or exceeds the permitted use, you will need to obtain permission directly from the copyright holder. To view a copy of this license, visit http://creativecommons.org/licenses/by/4.0/. 
often resembling processes seen in autoimmune diseases and possibly leading to permanent organ damage ${ }^{1-4}$.

Venous and arterial thromboembolism is pathologic formation of thrombi in organs, often associated with inflammation. Individuals with other chronic autoimmune disorders are known to be at risk for $\mathrm{TEE}^{5}$. Endothelial dysfunction and decreased thrombomodulindependent generation of activated protein $C$ have been implicated in GVHD pathogenesis, partially contributing to a procoagulant state ${ }^{6-9}$. Limited studies have reported a wide range of thromboembolism incidence among allogeneic HCT recipients ${ }^{10-13}$, with higher risk observed in patients developing $\mathrm{GVHD}^{12,13}$. Here, we aim to assess the incidence and risk factors for thromboembolic events (TEE), including venous thromboembolism (VTE) and pulmonary embolism (PE), among patients with known cGVHD and examine the impact of TEE on clinical outcomes after cGVHD.

\section{Methods}

\section{Study design and inclusion criteria}

The objective of this retrospective single-institution cohort study was to assess the incidence, risk factors, and clinical outcomes of patients with cGVHD who developed TEE. The study population included 145 consecutive adults who received their first allogeneic HCT and who developed cGVHD after a matched sibling (MSD) or umbilical cord blood (UCB) donor allogeneic HCT from 2010 to 2018 at the University of Minnesota. Bone marrow (BM), peripheral blood stem cell (PBSC), and UCB graft sources were included. Recipients received myeloablative (MAC) or reduced intensity conditioning (RIC) regimens. All GVHD prophylaxis strategies were included. All patients signed a written informed consent allowing the use of their medical data in clinical research analysis. This study was reviewed and approved by the University of Minnesota Institutional Review Board.

\section{Definitions}

Thromboembolic events (TEE), included venous thromboembolism (VTE) and pulmonary embolism (PE), and were defined as any new event, confirmed by imaging and requiring systemic therapy at any time after cGVHD diagnosis. We categorized VTE sites as upper extremity (UE) or lower extremity (LE) proximal or distal deep vein thrombosis (DVT). UE DVT included line associated events, central or peripherally inserted central catheter, and grouped separately for analysis.

We followed the 2014 NIH Consensus Criteria for diagnosis, determining organ involvement and overall severity at diagnosis of $\mathrm{CGVHD}^{14}$. cGVHD at onset was categorized as de-novo if cGVHD developed without prior acute GVHD (aGVHD), quiescent if cGVHD developed after resolution of prior aGVHD, and progressive if cGvHD developed without resolution of prior aGVHD.

Non-relapse mortality (NRM) was defined as death in the absence of disease relapse or progression, accounting for relapse as a competing risk. Overall survival (OS) was defined as time from transplantation to death from any cause.

\section{Patient and transplant characteristics}

The clinical factors examined for possible associations with TEE included: gender, age $(<50$ or $\geq 50)$, BMI $(<30$ or $\geq 30$ ), donor type (MSD or UCB), conditioning intensity (MAC vs RIC), GVHD prophylaxis (cyclosporine [CsA] or tacrolimus [Tac] with methotrexate [MTX], CsA or Tac or sirolimus [Siro] with mycophenolate mofetil [MMF]), disease risk index for malignant disorders (DRI: low risk, intermediate risk, high/very risk, non-malignant was not included) ${ }^{15}$, HCT comorbidity index ${ }^{16}$ (HCT-CI: low risk, intermediate risk, high risk), type of cGVHD at onset (denovo, quiescent or progressive), severity of cGVHD at onset (mild, moderate or severe), platelets at cGVHD diagnosis $(<50,000,50,000-100,000,>100,000)$, donorrecipient $\mathrm{ABO}$ match $(\mathrm{O}$ to $\mathrm{O}$ and non-O match groups for simplification given inclusion of double UCB), and cGVHD organ involvement (skin, eyes, mouth, joints, lung, gastrointestinal, genitourinary, liver). We additionally examined the effect of traditional TEE risk factors including smoking history, diabetes mellitus (DM), hyperlipidemia (HLD), hypertension (HTN), cerebrovascular accident (CVA), congestive heart failure (CHF), coronary artery disease (CAD), family history of TEE, and personal history of TEE prior to cGVHD diagnosis.

\section{Statistical analysis}

We assessed the cumulative incidence of TEE after cGVHD treating non-TEE mortality as a competing risk $^{17}$. Multivariate regression was used to evaluate the independent association of factors with the incidence of $\mathrm{TEE}^{18}$ using predetermined risk factors in our regression model including gender (male vs. female), age ( $<50$ vs. 250 ), severity of cGVHD at onset (mild vs. moderate vs. severe), $A B O$ blood group match ( $O$ to $O$ vs. non- $O$ match), history of CAD, and history of TEE prior to cGVHD. BMI and type of cGVHD (de-novo vs. quiescent vs. progressive) violated the proportional hazards assumption and were excluded from the regression model. Due to collinearity between organ involvement and cGVHD severity, only severity was included in the model. A separate model included cGVHD organ involvement at onset, mucocutanous (skin, oral and/or eye) vs. visceral. Given recurrent TEE episodes among some patients, the Prentice, Williams and Peterson model (PWP) for recurrent events was used to evaluate the 
independent association between the predetermined risk factors and $\mathrm{TEE}^{19}$. Cox and Fine and Gray regression models were used to evaluate the independent association of time-dependent VTE on OS and NRM, respectively, using propensity scoring to control for confounding ${ }^{18,20}$. Given the small number of events after censoring (30 for OS and 20 for NRM), analysis of the independent association of VTE on NRM and OS used a propensity score to control for confounding ${ }^{21}$.

All reported p-values were 2-sided. All analyses were performed using SAS 9.4 (SAS Institute, Inc., Cary, NC) and $\mathrm{R}$ version 3.6.2. Outcomes and covariates in regression models were all clinically pre-specified.

\section{Results}

Patient, treatment and cGVHD characteristics

Patient, disease, and transplant characteristics are shown in Table 1. A total of 145 patient who developed cGVHD were studied. Median age at time of cGVHD diagnosis was 52 years (range, 19-74). 104 (72\%) patients received MSD and $41(28 \%)$ single or double UCB allogeneic HCT. cGVHD was mild in severity in 24 patients $(16 \%)$, moderate in $82(57 \%)$, and severe in 39 (27\%). Type of cGVHD was de-novo in 55 patients (38\%), quiescent in 55 (38\%), and progressive in 35 (24\%). Patients developing TEE were more likely to have a history of CAD (44\% vs. $19 \%)$ and prior history of TEE before cGVHD diagnosis (32\% vs. $17 \%)$.

\section{TEE: incidence, subtype and timing after cGVHD}

TEE characteristics are shown in Table 2. Of the 145 patients with cGVHD, 32 (22\%) developed at least 1 TEE event, and 14 (10\%) developed 2 TEE events. No patients developed more than 2 TEE events. For the first TEE event, 6 patients developed a PE (19\%), 26 developed DVT (81\%), and 1 patient developed a thrombus in the inferior vena cava. Location of DVT was LE in 17 patients and UE in 8 patients, with 5 of these 8 UE DVTs catheter-related. For the second TEE events, 2 patients developed a PE (14\%), and 12 had a DVT (86\%; $n=5 \mathrm{LE}, n=7 \mathrm{UE}$, and $n=4$ of these 7 were catheter-related UE DVT). The cumulative incidence of TEE through 5 years post cGVHD diagnosis was estimated at 22\% (95\% CI, 15-29\%) with a median time from cGVHD to TEE of 234 days (range, 12-2050; interquartile range [IQR] 85-599). Median time to the development of LE DVT or PE was 107 days (range, 12-1925), and median time to development of UE DVT was 450 days (range, 158-1300).

Most patients were on corticosteroids at the time of first $(n=28,88 \%)$ and second $(n=10,71 \%)$ TEE, with a median prednisone dose equivalent to 0.3 and $0.2 \mathrm{mg} / \mathrm{kg} / \mathrm{day}$, respectively, averaged over 60 days prior to the TEE event. Sirolimus was the second most commonly used immunosuppression therapy (50 and 29\% at first and second TEE, respectively). IVIG (intravenous immunoglobulin) was
Table 1 Patient, disease and transplant characteristics.

\begin{tabular}{|c|c|}
\hline & All patients frequency (\%) \\
\hline$N$ & 145 \\
\hline Gender: Male & $95(66 \%)$ \\
\hline \multicolumn{2}{|l|}{ Age } \\
\hline Median(range), (IQR) & $52(19-74),(41-62)$ \\
\hline \multicolumn{2}{|l|}{ BMI } \\
\hline Median(range), (IQR) & 28.0 (19.2-50.2), (24.9-32.1) \\
\hline \multicolumn{2}{|l|}{ Donor Type } \\
\hline Matched Sibling & $104(72 \%)$ \\
\hline Single UCB & $9(6 \%)$ \\
\hline Double UCB & $32(22 \%)$ \\
\hline \multicolumn{2}{|l|}{ Conditioning } \\
\hline MAC & $63(43 \%)$ \\
\hline $\mathrm{RIC}$ & $82(57 \%)$ \\
\hline \multicolumn{2}{|l|}{ GVHD Prophylaxis } \\
\hline CsA or TAC/MTX & $51(35 \%)$ \\
\hline CsA or TAC/MMF & $86(59 \%)$ \\
\hline Siro/MMF & $8(6 \%)$ \\
\hline \multicolumn{2}{|l|}{ Diagnosis } \\
\hline Acute Leukemia & $86(60 \%)$ \\
\hline $\mathrm{CML} / \mathrm{CLL}$ & $6(4 \%)$ \\
\hline Lymphoma & $25(17 \%)$ \\
\hline Other & $28(19 \%)$ \\
\hline \multicolumn{2}{|l|}{ Karnofsky at HCT } \\
\hline$<90$ & $20(14 \%)$ \\
\hline$\geq 90$ & $125(86 \%)$ \\
\hline \multicolumn{2}{|l|}{$\mathrm{HCT}-\mathrm{Cl}$} \\
\hline Low Risk: 0 & 60 (41\%) \\
\hline Intermediate Risk: 1-2 & $42(29 \%)$ \\
\hline High Risk: $3+$ & $43(30 \%)$ \\
\hline \multicolumn{2}{|l|}{ ABO Recipient } \\
\hline A & $62(43 \%)$ \\
\hline B & $13(9 \%)$ \\
\hline$A B$ & $3(2 \%)$ \\
\hline O & $67(46 \%)$ \\
\hline \multicolumn{2}{|l|}{ ABO Donor } \\
\hline$A, B$ or $A B$ & 75 (52\%) \\
\hline $\mathrm{O}$ & 70 (48\%) \\
\hline \multicolumn{2}{|l|}{ Year of cGVHD } \\
\hline 2010-2013 & $76(52 \%)$ \\
\hline 2014-2018 & $69(48 \%)$ \\
\hline \multicolumn{2}{|l|}{ Days from $\mathrm{HCT}$ to $\mathrm{CGVHD}$} \\
\hline Median(range), (IQR) & $220(88-1111),(168-309)$ \\
\hline Karnofsky at cGVHD: $<90$ & $38(26 \%)$ \\
\hline \multicolumn{2}{|l|}{ cGVHD Type at Onset } \\
\hline De-novo & $55(38 \%)$ \\
\hline Quiescent & $55(38 \%)$ \\
\hline Progressive & $35(24 \%)$ \\
\hline \multicolumn{2}{|c|}{ cGVHD Global Severity at Onset } \\
\hline Mild & $24(16 \%)$ \\
\hline Moderate & $82(57 \%)$ \\
\hline Severe & $39(27 \%)$ \\
\hline \multicolumn{2}{|l|}{ Prior Acute GVHD Grade } \\
\hline No acute GVHD & $53(37 \%)$ \\
\hline Grade I-II & $50(34 \%)$ \\
\hline Grade III-IV & $42(29 \%)$ \\
\hline
\end{tabular}


Table 1 continued

\begin{tabular}{lc}
\hline & All patients frequency (\%) \\
\hline Number of Organs involved at cGVHD Onset & \\
1 or 2 & $75(52 \%)$ \\
$\geq 3$ & $70(48 \%)$ \\
WBC at cGVHD Diagnosis & \\
Median(range), (IQR) $\times 10^{9} / \mathrm{L}$ & $5.9(1.2-17.2),(4.3-7.9)$ \\
Platelets at CGVHD Diagnosis & \\
Median(range), (IQR) & $138(3-470),(99-183)$ \\
$<100,000 \times 10^{9} / \mathrm{L}$ & $38(26 \%)$ \\
$\geq 100,000 \times 10^{9} / \mathrm{L}$ & $107(74 \%)$ \\
cGVHD Organ Involvement & \\
Skin & $60(40 \%)$ \\
Eyes & $70(48 \%)$ \\
Mouth & $107(74 \%)$ \\
Liver & $42(29 \%)$ \\
Gastrointestinal & $59(41 \%)$ \\
Genitourinary & $7(5 \%)$ \\
Lung & $5(4 \%)$ \\
Joints & $15(10 \%)$ \\
CGVHD Treatment & \\
Systemic & $39(27 \%)$ \\
Topical & $12(8 \%)$ \\
Both & $94(65 \%)$ \\
\hline
\end{tabular}

$B M I$ body mass index, TEE thromboembolic events, CGVHD chronic graft-versushost disease, UCB umbilical cord blood, MAC myeloablative, RIC reduced intensity, CsA cyclosporine, Tac tarolimus, Siro sirolimus, MMF mycophenolate mofetil, MTX methotrexate, CML chronic myeloid leukemia, CLL Chronic lymphocytic leukemia, HCT hematopoietic cell transplantation.

administered within 60 days of TEE in 11 (34\%) and 3 (21\%) patients at the time of first and second TEE, respectively. At the time of TEE, 3 patients were receiving aspirin. 4 (13\%) and $8(57 \%)$ patients developed first and second TEE, respectively, on prophylactic anticoagulation (due to history of prior TEE). Enoxaparin was the most commonly used anticoagulation therapy. Figure 1 is a dot plot of TEE by anatomic location and time post HCT.

\section{Risk factors for the development of TEE}

Cumulative 5-year incidence of TEE was similar per gender ( $24 \%$ in males vs. $18 \%$ in females), age ( $23 \%$ vs. $21 \%$ with age $<50$ or $\geq 50)$, BMI ( $22 \%$ vs. $21 \%$ with $\mathrm{BMI}<30$ vs. $\geq 30$ ), donor source (23\% MSD vs. $20 \%$ UCB donor), conditioning ( $21 \%$ vs. $22 \%$ in MAC vs. RIC), GVHD prophylaxis $(22 \%$ for CsA or Tac/MTX vs. CsA or Tac or Siro/MMF), DRI (19\% in low and intermediate risk vs. 50\% in high/very high risk), HCT-comorbidity index (22\% in low and intermediate vs. $21 \%$ in high HCT-CI), or platelets at cGVHD diagnosis $(31 \%$ vs. $23 \%$ vs. $21 \%$ for counts of $<50,000$ vs. $50,000-100,000$ vs. $>100,000)$. TEE incidence was $25 \%$ in patients with de novo cGVHD $(95 \% \mathrm{CI}$, $13-37 \%$ ) compared to 20 and $17 \%$ in those with quiescent (95\% CI, 10-31\%) or progressive type (95\% CI, 5-30\%), respectively. Cumulative incidence was $9 \%(95 \% \mathrm{CI}$,
Table 2 Thromboembolism characteristics.

\begin{tabular}{|c|c|c|}
\hline & $\begin{array}{l}1^{\text {st }} \text { TEE } \\
\text { Frequency }(\%)\end{array}$ & $\begin{array}{l}2^{\text {nd }} \text { TEE } \\
\text { Frequency }(\%)\end{array}$ \\
\hline N & 32 & 14 \\
\hline $\begin{array}{l}\text { Median Days to Diagnosis of TEE from } \\
\text { cGVHD (Range), (IQR) }\end{array}$ & $\begin{array}{l}233.5(12-2050) \\
(84.5-599)\end{array}$ & $\begin{array}{l}715.5(19-2137) \\
(459-1139)\end{array}$ \\
\hline \multicolumn{3}{|l|}{ cGVHD Disease Status at TEE Event } \\
\hline Inactive & $5(16 \%)$ & $4(29 \%)$ \\
\hline Active & 27 (84\%) & $10(71 \%)$ \\
\hline \multicolumn{3}{|l|}{ Type and Location of TEE } \\
\hline PE & $6(19 \%)$ & $2(14 \%)$ \\
\hline DVT & $26(81 \%)$ & $12(86 \%)$ \\
\hline LE Left & 9 & 3 \\
\hline LE Right & 7 & 2 \\
\hline LE Bilateral & 1 & 0 \\
\hline UE Left & 4 & 2 \\
\hline UE Right & 4 & 5 \\
\hline IVC & 1 & 0 \\
\hline Occlusive DVT & $16(61 \%)$ & $4(33 \%)$ \\
\hline UE Catheter Related DVT & $5(19 \%)$ & $4(33 \%)$ \\
\hline \multicolumn{3}{|l|}{ Lab values at TEE Diagnosis } \\
\hline \multicolumn{3}{|l|}{ Median (range), (IQR) } \\
\hline WBC $\left(\times 10^{9} / \mathrm{L}\right)$ & $\begin{array}{l}7.1(0.6-21.2) \\
(4.3-11.2)\end{array}$ & $\begin{array}{l}6.7(3.3-11.1) \\
(5.2-9.3)\end{array}$ \\
\hline Absolute neutrophil count $\left(\times 10^{9} / \mathrm{L}\right)$ & $\begin{array}{l}4.3(0.6-14.6) \\
(3.5-7.6)\end{array}$ & $\begin{array}{l}4.3(1.4-9.2) \\
(3.5-7.2)\end{array}$ \\
\hline Absolute lymphocyte count $\left(\times 10^{9} / \mathrm{L}\right)$ & $\begin{array}{l}1.0(0-5.2) \\
(0.5-1.8)\end{array}$ & $\begin{array}{l}1.3(0.3-9.5) \\
(0.9-1.6)\end{array}$ \\
\hline Absolute eosinophil count $\left(\times 10^{9} / \mathrm{L}\right)$ & $0(0-5.4),(0-0.1)$ & $0.1(0-0.7),(0-0.3)$ \\
\hline Hemoglobin mg/dl & $\begin{array}{l}11.2(7.4-15.3) \\
(9.9-13.0)\end{array}$ & $\begin{array}{l}12.0(8.7-16.1) \\
(10.5-14.4)\end{array}$ \\
\hline Platelet $\left(\times 10^{9} / \mathrm{L}\right)$ & $\begin{array}{l}112(8-346) \\
(57-174)\end{array}$ & $\begin{array}{l}136(38-326) \\
(61-184)\end{array}$ \\
\hline Lactate dehydrogenase $\mathrm{U} / \mathrm{L}$ & $\begin{array}{l}437(98-1122) \\
(311-806)\end{array}$ & $384(311-457)$ \\
\hline Albumin & $\begin{array}{l}3.2(1.5-4.2) \\
(2.7-3.5)\end{array}$ & $\begin{array}{l}3.1(1.6-3.6) \\
(2.5-3.4)\end{array}$ \\
\hline
\end{tabular}

Immunosuppression Medications at and 60 days prior to TEE Number (proportion)

\begin{tabular}{lll} 
Corticosteroids & $28(88 \%)$ & $10(71 \%)$ \\
Prednisone Dose equivalent mg/kg/ & $0.3(0.1-1.64)$, & $0.2(0.04-1.6)$, \\
day (averaged over 60 days prior to & $(0.2-0.5)$ & $(0.07-0.31)$ \\
TEE); Median (range), (IQR) & & \\
Budesonide & $3(9 \%)$ & $2(14 \%)$ \\
Sirolimus & $16(50 \%)$ & $4(29 \%)$ \\
MMF & $1(3 \%)$ & 0 \\
Rituximab & $2(6 \%)$ & 0 \\
CNI & $5(16 \%)$ & $2(14 \%)$ \\
TNF-inhibitor & $1(3 \%)$ & $1(7 \%)$ \\
ECP & $2(6 \%)$ & $1(7 \%)$ \\
IVIG & $11(34 \%)$ & $3(21 \%)$ \\
Immunomodulatory Drugs & $1(3 \%)$ & 0 \\
Erythropoietin/Thrombopoietin & $2(6 \%)$ & 0 \\
Ibrutinib or ruxolitinib & $2(6 \%)$ & $3(21 \%)$ \\
Antiplatelet therapy (Aspirin) & $3(9 \%)$ & $3(21 \%)$ \\
\hline
\end{tabular}

*Duration expressed in median (range), (interquartile range).

cGVHD chronic graft-versus-host disease, TEE thromboembolic events, $P E$ pulmonary embolism, DVT deep vein thrombosis, $L E$ lower extremity, UE upper extremity, IVC inferior vena cava, MMF mycophenolate mofetil, CNI calcineurin inhibitor, TNF tumor necrosis factor, ECP extracorporeal photopheresis, IVIG intravenous immunoglobulin. 


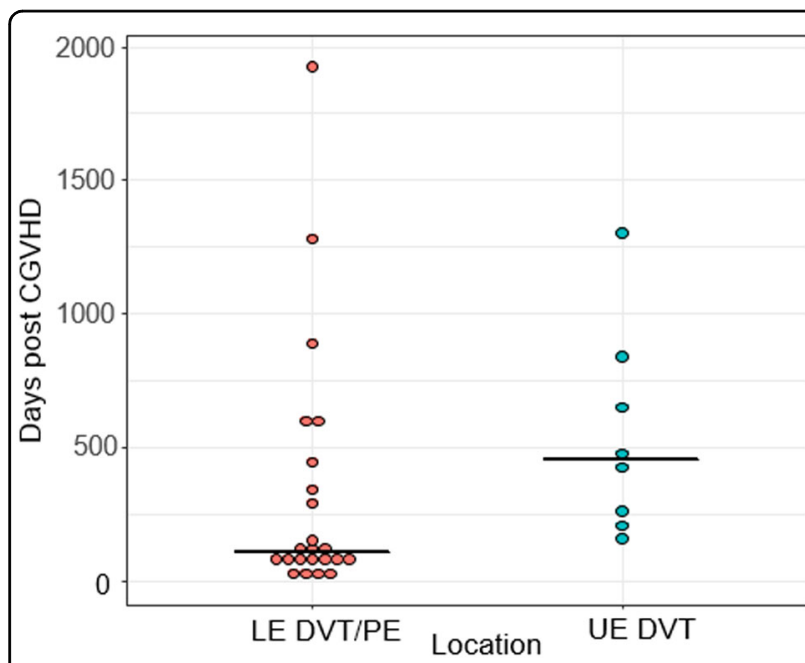

Fig. 1 Dot plot of thromboembolic events. Each dot represents a unique event. Lower extremity (LE) deep vein thrombosis (DVT) events are displayed with pulmonary embolism (PE) events on the left. Upper extremity (UE) DVT events are displayed on the right.

0-20\%), 17\% (95\% CI, 9-25\%), and 38\% (95\% CI, 22-55\%) in those with mild, moderate, and severe GVHD, respectively. When we examined the incidence per organ involvement, patients with lung cGVHD had a higher 5year cumulative incidence TEE (60\%, 95\% CI [27-93\%] vs. $20 \%$, CI [13-27]). Donor-recipient ABO blood group O-O had lower 5 -year cumulative incidence of TEE (11\%; 95\% CI, 1-22) compared to all other ABO matches combined (26\%; 95\% CI, 17-35).

In multivariate regression, we examined the independent impact of clinical factors on the development of TEE using the stated predetermined risk factors (Fig. 2). Higher risk for TEE was associated with cGVHD severity (hazard ratio $[\mathrm{HR}] 4.9$, [95\% CI, 1.1-22.0]; $p=0.03$ ), non$\mathrm{O}$-donor to recipient $\mathrm{ABO}$ match compared to O-donor to O-recipient match (HR 2.7, [95\% CI, 1.0-7.5]; $p=$ 0.053 ), and personal history of coronary artery disease (HR 2.4, [95\% CI, 1.1-5.3]; $p=0.03$ ). Organ involvement at cGVHD onset (mucocutanous vs. visceral) was not associated with risk of subsequent TEE.

\section{TEE effect on non-relapse mortality and survival}

TEE was not associated with 2-year NRM (HR 1.2, [95\% CI; 0.4-3.6]) or 5-year OS (HR 1.4, [95\% CI, 0.7-3.0]). The 5 -year NRM and OS were not significantly different per TEE location. Disease relapse was the predominant cause of death in $55 \%$ of patients developing TEE compared to $34 \%$ in those without TEE. Relapses occurred in 5 patients before TEE diagnosis and in 7 patients after TEE with median time to relapse 302 days (range, 128-1231) after TEE. cGVHD was the primary cause of death in $11 \%$ of those developing TEE compared to $20 \%$ in those without TEE.

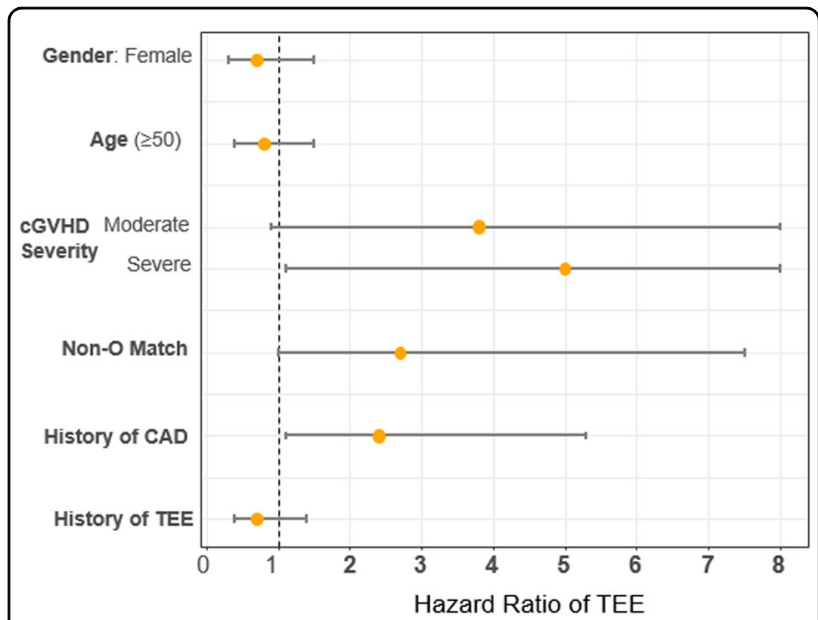

Fig. 2 Multivariate regression of risk factors for thromboembolic event development among all patients with cGVHD. $\mathrm{CGVHD}=$ chronic graft-versus-host disease; $C A D=$ coronary artery disease. Non$\mathrm{O}$ match $=$ includes all donor-recipient $\mathrm{ABO}$ match other than $\mathrm{O}$ to $\mathrm{O}$.

\section{Discussion}

cGVHD is a complication after allogeneic HCT that is associated with high risk for developing thromboembolic complications. The risk of developing LE DVT or PE occur earlier than UE DVT; however, both can occur years after cGVHD diagnosis and require high levels of clinical attention. Our study showed that cGVHD severity, non-O donor-recipient $\mathrm{ABO}$ group match, and personal history of CAD are associated with higher risk of TEE development after cGVHD.

cGVHD is a cytokine-driven and immune-mediated complication resulting in systemic inflammation and endothelial dysfunction. Endothelial structure and function are central to orchestrating inflammatory and thrombotic responses. Cytotoxic T-lymphocytes and inflammatory cytokines contribute to endothelial injury after allogeneic $\mathrm{HCT}$, which in turn can lead to impaired tissue perfusion and fibrosis $3,22,23$. Evidence of endothelial activation and damage can be found early post transplantation and is central to GVHD and other endothelial-driven complications after allogeneic $\mathrm{HCT}^{2,24-26}$. Additionally, further understanding of altered primary and secondary hemostasis after cGVHD will be critical to balance the increased risk of bleeding with the benefit of thromboprophylaxis in this population ${ }^{10,12}$.

There is a well-known association between $\mathrm{ABO}$ blood group and risk of thrombosis; particularly, those with a non-O blood group are at higher risk of arterial and venous thrombosis ${ }^{27-29}$. This increased risk is partially due to qualitative and quantitative differences in the glycoprotein von Willebrand factor (vWF), including 25\% higher plasma levels of vWF in non-O blood group individuals $^{30,31}$. VWF is not required for retention of red 
blood cells in clots ${ }^{32}$. However, ABO blood group contributes to VWF proteolysis and clearance and may contribute to VWF interactions with platelet glycoprotein IbIX-V and glycoprotein IIb-IIa complexes on platelet surfaces $^{33-35}$. Other potential mechanisms for increased TEE post-transplant may include hemolysis, increased transfusions secondary to delayed RBC engraftment, and changes in endothelial cells ${ }^{36}$. In our cohort, we identified non-O donor-recipient group as an independent risk factor for TEE development after cGVHD compared to the O-O ABO group. This association has not been previously reported and warrants further investigation of $\mathrm{ABO}$ blood group effect on risk of thrombosis after allogeneic $\mathrm{HCT}$, specifically in high-risk patients.

Our study identified a subgroup of allogeneic HCT recipients at a high risk for TEE. If this subgroup of allogeneic HCT recipient could be identified prior to the development of TEE, they could be treated with early thromboprophylaxis and other supportive care strategies for prevention of TEE. Biomarkers of endothelial dysfunction after cGVHD could identify the subgroup of allogeneic HCT recipients at risk of developing TEE that would benefit most from intervention.

\section{Acknowledgements}

This research was supported by NIH grant P30 CA77598 utilizing the Biostatistics Core of the Masonic Cancer Center, University of Minnesota and the National Center for Advancing Translational Sciences of the National Institutes of Health Award Number UL1TR002494. We would like to acknowledge Michael Franklin, MS, for assistance in editing this manuscript.

\section{Author details}

${ }^{1}$ Blood and Marrow Transplant Program, Department of Medicine, University of Minnesota, Minneapolis, MN, USA. ²Division of Hematology, Oncology, and Transplantation, Department of Medicine, University of Minnesota, Minneapolis, MN, USA. ${ }^{3}$ Biostatistics and Informatics, Clinical and Translational Science Institute, University of Minnesota, Minneapolis, MN, USA. ${ }^{4}$ Department of Pediatrics, University of Alabama, Tuscaloosa, AL, USA

\section{Conflict of interest}

D.J.W.-research support from Incyte. B.R.B.-founder of Tmunity Therapeutics, advisory board member for Kadmon Pharmaceuticals, Magenta Therapeutics, and BlueRock Therapeutics and receives research funding from BlueRock Therapeutics. S.G.H.- consultant for Incyte. The remaining authors declare no competing interests.

\section{Publisher's note}

Springer Nature remains neutral with regard to jurisdictional claims in published maps and institutional affiliations.

Supplementary information The online version contains supplementary material available at https://doi.org/10.1038/s41408-021-00488-2.

Received: 15 March 2021 Revised: 20 April 2021 Accepted: 29 April 2021 Published online: 18 May 2021

\footnotetext{
References

1. MacDonald, K. P. A., Blazar, B. R. \& Hill, G. R. Cytokine mediators of chronic graftversus-host disease. J. Clin. Invest. 127, 2452-2463 (2017).
}

2. Furukawa, M. et al. A critical role of the Gas6-Mer axis in endothelial dysfunction contributing to TA-TMA associated with GVHD. Blood Adv. 3 2128-2143 (2019).

3. Luft, T. et al. Steroid-refractory GVHD: T-cell attack within a vulnerable endothelial system. Blood 118, 1685-1692 (2011).

4. Dietrich, S. et al. Endothelial vulnerability and endothelial damage are associated with risk of graft-versus-host disease and response to steroid treatment. Biol. Blood Marrow Transplant. 19, 22-27 (2013).

5. Zöller, S. B. et al. Articles Risk of pulmonary embolism in patients with autoimmune disorders: a nationwide follow-up study from Sweden. Lancet 379, 244-293 (2012)

6. Mosnier, L. O., Yang, X. V. \& Griffin, J. H. Activated protein C mutant with minimal anticoagulant activity, normal cytoprotective activity, and preservation of thrombin activable fibrinolysis inhibitor-dependent cytoprotective functions. J. Biol. Chem. 282, 33022-33033 (2007).

7. Griffin, J. H., Zlokovic, B. V. \& Mosnier, L. O. Activated protein C: biased for translation. Blood 125, 2898-2907 (2015).

8. Ranjan, S. et al. Activated protein C protects from GvHD via PAR2/ PAR3 signalling in regulatory T-cells. Nat. Commun. 8, 1-16 (2017).

9. Ikezoe, T., Yang, J., Nishioka, C. \& Yokoyama, A. Thrombomodulin alleviates murine GVHD in association with an increase in the proportion of regulatory T cells in the spleen. Bone Marrow Transplant. 50, 113-120 (2015).

10. Gerber, D. E. et al. The incidence of and risk factors for venous thromboembolism (VTE) and bleeding among 1514 patients undergoing hematopoietic stem cell transplantation: implications for VTE prevention. (2008). https://doi. org/10.1182/blood-2007-10-117051.

11. Gonsalves, A. et al. Incidence of symptomatic venous thromboembolism following hematopoietic stem cell transplantation. J. Thromb. Haemost. 6, 1468-1473 (2008).

12. Labrador, J. et al. Analysis of incidence, risk factors and clinical outcome of thromboembolic and bleeding events in 431 allogeneic hematopoietic stem cell transplantation recipients. Haematologica 98, 437-443 (2013).

13. Kekre, N. et al. Venous thromboembolism is associated with graft-versus-host disease and increased non-relapse mortality after allogeneic hematopoietic stem cell transplantation. Haematologica 102, 1185-1191 (2017).

14. Jagasia, M. H. et al. Nih GVHD 2014. 21, 389-401 (2016).

15. Armand, P. et al. Validation and refinement of the disease risk index for allogeneic stem cell transplantation. Blood 123, 3664-3671 (2014).

16. Sorror, M. L. et al. Hematopoietic cell transplantation (HCT)-specific comorbidity index: a new tool for risk assessment before allogeneic HCT. Blood 106, 2912-2919 (2005)

17. Lin, D. Y. Non-parametric inference for cumulative incidence functions in competing risks studies. Stat. Med. 16, $901-910$ (1997).

18. Fine, J. P. \& Gray, R. J. A proportional hazards model for the subdistribution of a competing risk. J. Am. Stat. Assoc. 94, 496-509 (1999).

19. Prentice, R. L., Williams, B. J. \& Peterson, A. V. On the regression analysis of multivariate failure time data. Biometrika 68, 373-379 (1981).

20. Cox, D. R. Regression models and life-tables. J. R. Stat. Soc. Ser. B 34, (1972).

21. Rosenbaum, P. R. \& Rubin, D. B. The central role of the propensity score in observational studies for causal effects. Matched Sampl. Causal Eff. 170-184 (2006). https://doi.org/10.1017/CBO9780511810725.016.

22. Biedermann, B. C. et al. Endothelial injury mediated by cytotoxic T lymphocytes and loss of microvessels in chronic graft versus host disease. Lancet 359, 2078-2083 (2002)

23. Bouazzaoui, A. et al. Endothelial dysfunction and altered mechanical and structural properties of resistance arteries in a murine model of graft-versushost disease. Biol. Blood Marrow Transplant. 20, 1493-1500 (2014).

24. Salat, C. et al. Endothelial cell markers in bone marrow transplant recipients with and without acute graft-versus-host disease. Bone Marrow Transplant 19, 909-914 (1997)

25. Gloude, N. J. et al. Circulating dsDNA, endothelial injury, and complement activation in thrombotic microangiopathy and GVHD. Blood 130, 1259-1266 (2017).

26. Luzzatto, G. et al. Markers of endothelial function in pediatric stem cell transplantation for acute leukemia. Med. Pediatr. Oncol. 40, 9-12 (2003).

27. $\mathrm{Wu}, \mathrm{O} .$, Bayoumi, N., Vickers, M. A. \& Clark, P. ABO(H) blood groups and vascular disease: a systematic review and meta-analysis. J. Thrombosis Haemost. $\mathbf{6}$, 62-69 (2008).

28. Ketch, T. R. et al. ABO blood types: Influence on infarct size, procedural characteristics and prognosis. Thromb. Res. 123, 200-205 (2008). 
29. Wang, G. et al. Association between ABO blood group and venous thrombosis related to the peripherally inserted central catheters in cancer patients. J. Vasc. Access (2020). https://doi.org/10.1177/1129729820954721.

30. Jenkins, P. V. \& O'Donnell, J. S. ABO blood group determines plasma von Willebrand factor levels: a biologic function after all? Transfusion 46 1836-1844 (2006)

31. Lenting, P. J., Christophe, O. D. \& Denis, C. V. Von Willebrand factor biosynthesis, secretion, and clearance: connecting the far ends. Blood $\mathbf{1 2 5}$ 2019-2028 (2015).

32. Holle, L. A., Monroe, D. M. \& Wolberg, A. S. WF (von Willebrand Factor) is not required for red blood cell retention in clots in mice. Arterioscler. Thromb. Vasc. Biol. 40, 1952-1954 (2020).
33. Ward, S., O'Sullivan, J. \& O'Donnell, J. S. The relationship between ABO blood group, von Willebrand factor and primary hemostasis. Blood (2020). https://doi. org/10.1182/blood.2020005843.

34. Brophy, T. M. et al. Plasmin cleaves von willebrand factor at K1491-R1492 in the A1-A2 linker region in a shear- and glycan-dependent manner in vitro. Arterioscler. Thromb. Vasc. Biol. 37, 845-855 (2017).

35. Albánez, S. et al. Aging and ABO blood type influence von Willebrand factor and factor VIII levels through interrelated mechanisms. J. Thromb. Haemost. 14 953-963 (2016).

36. Lin, Y., Weisdorf, D. J., Solovey, A. \& Hebbel, R. P. Origins of circulating endothelial cells and endothelial outgrowth from blood. J. Clin. Invest. 105, 71-77 (2000). 